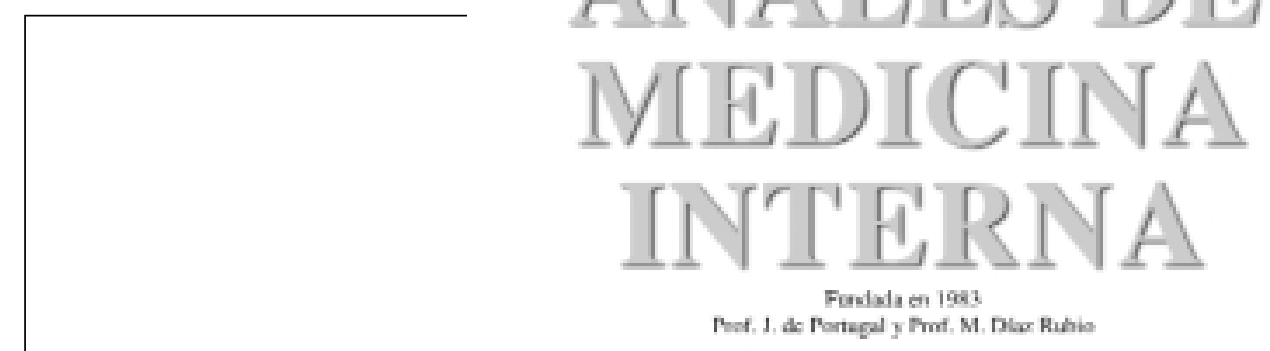

Cartas al

\section{Coma e insuficiencia respiratoria tras fractura de fémur y tibia}

\section{Sr. Director:}

Varón de 19 años sin antecedentes patológicos de interés que acude al servicio de Urgencias tras accidente de tráfico presentando fractura de tibia y fémur derechos. No ha sufrido traumatismo craneoencefálico. Al ingreso se encuentra hemodinámicamente estable con una saturación de oxígeno basal del $99 \%$ y presenta un Glasgow de 15 con una exploración neurológica normal.

Cuatro horas más tarde presenta un cuadro de inicio brusco de desorientación, obnubilación y taquipnea permaneciendo la exploración neurológica normal con cifras tensionales mantenidas, aunque con caída de la saturación de oxígeno a 92\%, por lo que se realizaron una radiografía de tórax y un TAC craneal que fueron normales. A las 8 horas del traumatismo se realiza intervención quirúrgica colocando sendos clavos de Gross en tibia y fémur. Tras pasar a planta el paciente se encuentra estuporoso, taquicárdico, taquipneico con tensión arterial de 100/50 mm Hg por lo que es trasladado a la Unidad de Cuidados Intensivos presentando tetraparesia y disminución progresiva del nivel de conciencia hasta entrar en estado de coma y empeoramiento del compromiso respiratorio que requiere conexión a ventilación mecánica. En este momento se realiza un nuevo TAC craneal que vuelve a ser normal. En la analítica de sangre destacan una hemoglobina de $8,7 \mathrm{~g} / \mathrm{l}$, hematocrito $24,6 \%$, plaquetas $145.000 / \mathrm{mm}^{3}$, sin alteraciones bioquímicas. A las 24 horas presenta erupción petequial localizada en región anterior de tórax, axilas y cuello. La radiografía de tórax no muestra hallazgos patológicos y se realiza un electroencefalograma que sugiere lesiones frontales corticales y afectación mesencefálica, así como potenciales evocados compatibles con afectación a nivel de tronco cerebral izquierdo. En el examen de fondo de ojo aparecen áreas sugestivas de isquemia retiniana. Se realiza una resonancia magnética (RMN) cerebral en la que se aprecian múltiples imágenes hiperintensas difusas en sustancia blanca tanto a nivel supratentorial como a nivel de tronco, cuerpo calloso y pedúnculos cerebelosos. (Fig. 1).

Con el diagnóstico de embolia grasa cerebral se mantuvieron medidas de soporte, retirando la ventilación mecánica tras 14 días presentando respiración espontánea con mejoría progresiva del nivel de conciencia.

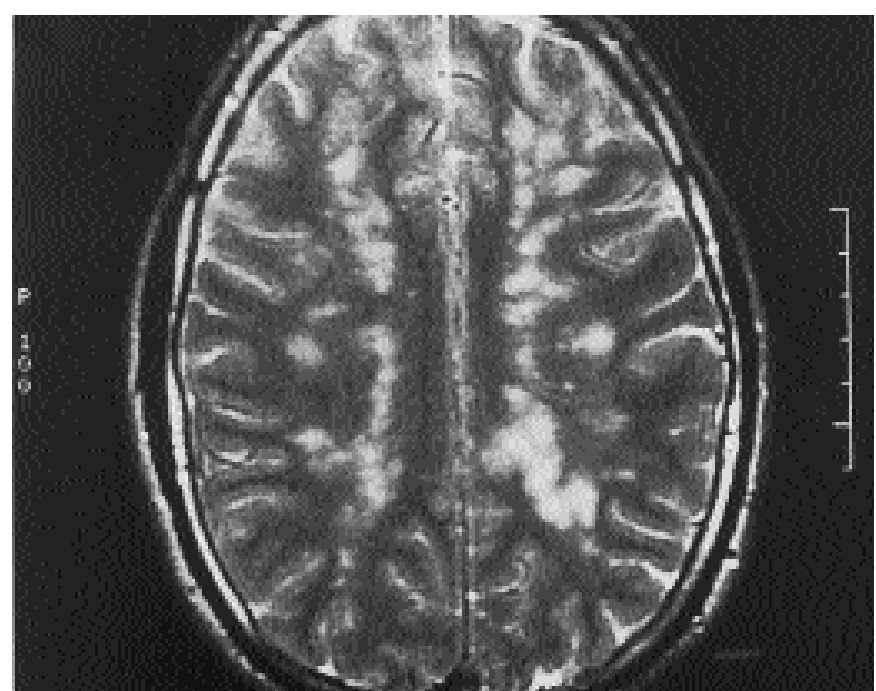

Fig. 1. Corte horizontal de RMN cerebral apreciándose lesiones hiperintensas difusas.

$\mathrm{Al}$ cabo de dos meses presenta una puntuación de 15 en la escala de Glasgow y ha recuperado de forma marcada la movilidad en extremidades.

El síndrome de embolia grasa cerebral se presenta en el 1$5 \%$ de las fracturas de huesos largos y de forma más rara se asocia a cirugía de la obesidad, diabetes mellitus, pancreatitis, esteatosis hepática, quemaduras graves y tratamiento esteroideo (1).

Su patogenia se explica por la embolización de partículas grasas que alcanzan primariamente los capilares pulmonares para posteriormente producir una embolización sistémica principalmente hacia el cerebro y riñón. A estos niveles el daño es producido por un mecanismo obstructivo que conlleva una isquemia cerebral y por un mecanismo tóxico (2). Hay algún caso publicado asociado a foramen oval permeable (3).

Suele manifestarse clínicamente habitualmente a las 24-72 horas posteriores al traumatismo, aunque hay formas fulminantes que aparecen en las primeras 12 horas.

El diagnóstico es fundamentalmente clínico destacando la tria- 
da clásica de hipoxemia, alteraciones neurológicas (encefalopatía difusa con disminución del nivel de conciencia o afectación focal) y erupción petequial. Otros síntomas menores son taquicardia, trombopenia, fiebre, glóbulos grasos en orina o esputo, embolia retiniana y caída del hematocrito (4).

El TAC cerebral puede ser normal o mostrar inicialmente áreas de alta atenuación difusas y edema cerebral y posteriormente áreas hipodensas y atrofia cerebral. La RMN cerebral parece una exploración más sensible para detectar lesiones en el síndrome de embolismo graso, presentando típicamente áreas puntiformes de baja señal en T1 y de alta señal en T2 con predominio en zonas profundas de la sustancia blanca $(1,5)$. Se remienda su uso en aquellos casos en los que el TAC es normal y la clínica no es característica. El SPECT puede mostrar una disminución de flujo a nivel cerebral (2).

El tratamiento incluye medidas de soporte con sueroterapia y soporte ventilatorio en los casos más severos. El uso de corticoides es controvertido, al igual que el periodo de corrección de la fractura, aunque parece que la fijación en las primeras 24 horas disminuye la incidencia de este síndrome. Su mortalidad es de aproximadamente el 5\%, aunque en la mayoría de los casos la recuperación es completa.

\section{Repiso Moreno, J. L. Peña Somovilla, A. Moreno Ortigo- $\mathbf{s a}^{1}$, F. Jiménez Bermejo, $M^{\mathbf{a}}$ E. Marzo Sola ${ }^{2}$}

Servicio de Medicina Interna. ${ }^{1}$ Unidad de Medicina Intensiva. ${ }^{2}$ Sección de Neurología. Complejo Hospitalario San Millán-San Pedro. Logroño

1. Guedea A, Barrena R, Guelbenzu S, Tejada A. Embolia grasa cerebral: valoración mediante resonancia magnética. Radiología 1998; 40 (2): 125-42.

2. Satoh H, Kurisu K, Ohtani M, Arita K, Okabayashi S, Nakahara T et al. Cerebral fat embolism studied by magnetic resonance imaging, transcranial doppler sonography and single foton emission computed tomography: case report. J Trauma 1997; 43 (2): 345-8.

3. Etchells E, Wong D, Davidson G, Houston P. Fatal cerebral fat embolism associated with a patent foramen ovale. Chest 1993; 104 (3): 962-3.

4. Gurd AR. Fat embolism: an aid to diagnosis. J Bone Surg 1970; 52 B: 732-7.

5. Yoshida A, Okada Y, Nagata Y, Hanaguri K, Morio M. Assesment of cerebral fat embolism by magnetic resonance imaging in the acute stage. J Trauma 1996, 40 (3): 436-40.

\section{Hematoma retroperitoneal espontáneo inducido por enoxiparina a dosis terapéuticas}

\section{Sr. Director:}

La patología del espacio retroperitoneal tiene gran interés tanto por el compromiso que puede representar para los órganos allí situados como por la dificultad de diagnóstico diferencial. Dentro de las patologías del retroperitoneo destaca por su rareza el hematoma retroperitoneal espontáneo en pacientes anticoagulados, al presentarse como abdomen agudo (1). El hematoma retroperitoneal asociado a enoxiparina a dosis terapéuticas no es un hecho común, ya que en la búsqueda MEDLINE desde enero 1995 hasta abril 2002, sólo hemos encontrado la comunicación de dos casos asociados a la utilización de enoxiparina a dosis de $1 \mathrm{mg} / \mathrm{kg} / 12 \mathrm{~h}(1,2)$.

Caso: Mujer de 68 años, alérgica a penicilina, hipertensa desde por lo menos 20 años y con insuficiencia renal crónica por nefroangioesclerosis, esplenectomizada desde hace 10 años por trombosis esplénica, obstrucción ileo-femoral 6 años antes del ingreso, realizando tromboembolectomía, realizado estudio de hipercoagulabilidad tres años antes del ingreso con resistencia a la proteína $\mathrm{C}$ activada y niveles elevados de factor VIII, junto con anticuerpos anticardiolipina, por lo que se recomienda tratamiento con anticoagulación oral. Ingreso por sepsis de foco urológico por morganella morganii, retirando acenocumarol en el contexto de tratamiento de sepsis, al cuarto día de su estancia en planta y estando en tratamiento con enoxiparina a dosis terapéuticas, $60 \mathrm{mg}$ cada 12 horas $(1 \mathrm{mg} / \mathrm{kg} / 12 \mathrm{~h})$, presenta intenso dolor abdominal con irradiación hacia fosa renal e iliaca izquierdas, y desarrollo posterior de ileo abdominal además de dolor de características neuropáticas en pierna izquierda, se sigue de anemización hasta 2,79 milimoles/L hemoglobina $(4,5 \mathrm{~g} / \mathrm{dl})$ deterioro de la función renal 213,5 micromoles $/ 1$ (2,8 mg/dl) creatinina. Ante el deterioro clínico y hemodinámico se procedió a la realización de TAC abdominal con contraste oral y endovenoso que muestra colección retroperitoneal izquierda con realce de contraste periférico que se confunde con el psoas y presenta falta de definición con la grasa vecina, con extensión a pelvis y fosa iliaca izquierda. Para descartar causa estructural vascular que pudiera explicar el sangrado retroperitoneal se completaron los estudios de imagen con angioresonancia de abdomen que confirma gran masa retropeitoneal izquierda, que comienza a visualizarse desde la altura del riñón y que termina en pelvis, desplazando el polo superior del riñón en sentido medial y anterior estableciendo simultáneamente contacto con la iliaca izquierda. La enferma evolucionó satisfactoriamente con tratamiento conservador: administración de cuatro concentrados de hematíes, medidas de soporte, que incluyeron retirada de enoxiparina. No se pudo determinar la actividad anti factor Xa. La enoxiparina a dosis terapéuticas fue administrada durante 6 días.

Discusión: Las heparinas de bajo peso molecular (HBPM) han demostrado ser tan seguras y efectivas como las heparinas no fraccionadas en el tratamiento de la trombosis venosa profunda (TVP) y tromboembolismo pulmonar agudo (TEPA), y en profilaxis de fenómenos tromboembólicos $(1,3,4)$. Por no necesitar habitualmente monitorización, y por su facilidad de administración ambulatoria, se ha ido cambiando la práctica clínica en el terreno de la anticoagulación $(5,6)$.

Las HBPM son fragmentos de la heparina no fraccionada con un peso molecular de 5000 daltons, con mecanismo de acción diferente al de las heparinas no fraccionadas, al inhibir la actividad del factor Xa y de la trombina. Además las HBPM producen una respuesta anticoagulante muy predecible que se explica por su mejor biodisponibilidad, mayor vida media y cinética de aclaramiento dosis dependiente, por lo que no suele ser necesaria la monitorización de estudios de coagulación (3). Los factores de riesgo para sangrado con la utilización de HBPM son la insuficiencia renal y la edad avanzada (3). La insuficiencia renal prolonga la eliminación de enoxiparina, aunque no se ha encontrado relación lineal con el aclaramiento de creatinina (7).

La incidencia de complicaciones hemorrágicas asociadas a la utilización de HBPM no se conoce con exactitud al ser descritos únicamente casos aislados de hemorragia intrahepática, hematomas del psoas, pared abdominal, retroperitoneal y epidural, éste último tras procedimientos de anestesia epidural $(8,9)$.

El retroperitoneo es un espacio virtual que puede convertirse en un reservorio de gran cantidad de sangre, lo cual se traduce en una falta inicial de sintomatología clínica hasta la aparición de un cuadro de hipovolemia (10).

La situación clínica ideal sería la existencia de dolor abdominal junto con la presencia de una masa o empastamiento abdominal asociado a un descenso del hematocrito, como, ocurrió en el caso presentado, siendo de destacar la presencia de distensión abdominal como signo de ileo paralítico subyacente al propio hematoma retroperitoneal. 